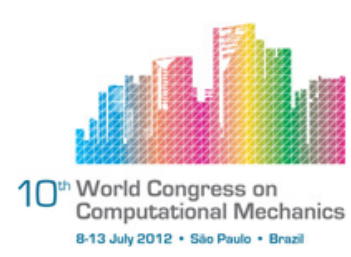

\title{
RECONSTRUCTION OF CHLOROPHYLL-A VERTICAL PROFILES WITH ENHANCED RESOLUTION
}

\author{
R. P. Souto ${ }^{1}$, P. L. S. Dias ${ }^{1}$, H. F. Campos Velho' ${ }^{2}$, S. Stepnhany ${ }^{2}$, M. Kampel ${ }^{3}$ \\ ${ }^{1}$ National Laboratory for Scientific Computing (rpsouto@lncc.br) \\ ${ }^{2}$ Associated Laboratory of Computing and Applied Math at National Institute for Space Re- \\ search
}

${ }^{3}$ Department of Remote Sensing at National Institute for Space Research

\begin{abstract}
We present a methodology to reconstruct vertical profiles of Chlorophyll-a pigment concentration in open-ocean waters based on radiance values at different depths. The inverse problem is formulated here as an optimization problem and iteratively solved by an Ant Colony System (ACS) meta-heuristic. An objective function is given by the square difference between computed and experimental radiances at each iteration. The Laplace transform discrete ordinate (LTSN) method is used to solve the radiative transfer equation (direct problem) in order to compute the radiances. As radiance intensity decays exponentially with depth, a correction factor must be employed to weight the influence of the depth-dependent radiances. We previously proposed a correction factor that is given by the ratio between the mean radiance - related to the polar angle - at each level and the mean radiance at the surface. Here we obtained results close to the exact solution even for noisy radiance data, especially for smooth profiles, where the smoothness is determined by the second order Tikhonov criterion. However, for profiles with two or more peaks of Chlorophyll-a concentration, the methodology is still not able to identify these extremes. This result can be explained partly by the relatively low number of sampling points (usually 10 points), that limits the geometric resolution of the vertical profile to be reconstructed. Alternatively, we propose in this work the reconstruction vertical profile using a higher geometric resolution. We use at least one meter between each recovered point in order to evaluate the ability of identifying any peak in Chlorophyll-a concentration. Two inversion strategies are evaluated. At first, the resolution of the vertical profile is increased with a greater number of points to be recovered. In the second strategy, a hybrid methodology is used: initially the original methodology is employed, using the ACS stochastic method to retrieve a low resolution profile (10 points), and then, this result is used as an initial guess for the deterministic method of optimization Levenberg-Marquardt to retrieve a higher resolution profile (with at least 1 point at each meter depth). A comparison between these two strategies is presented and discussed.
\end{abstract}

Keywords: Inverse Problems, Hydrologic Optics, Ant Colony System, Levenberg-Marquardt. 


\section{INTRODUCTION}

The direct or forward radiative transfer problem in hydrologic optics, in the steady state, involves the determination of the radiance distribution in a body of water, given the boundary conditions, source term, inherent optical properties (IOPs), as the absorption $a$ and scattering $b$ coefficients, and the scattering phase function.

The corresponding inverse radiative transfer problem arises when physical properties, internal light sources and/or boundary conditions must be estimated from radiometric measurements of the underwater light field.

The radiance values near the surface are much higher than those in deeper water, since radiance value decays nearly exponentially with depth. A depth correction factor for the radiances was first proposed by [12]. This work employs a correction factor based on the mean radiance at each depth level/region [11].

In this work, the inverse model is an implicit technique for function estimation from synthetic radiometric measurements in several depths and single wavelength. The algorithm is formulated as a constrained nonlinear optimization problem, in which the direct problem is iteratively solved for successive approximations of the unknown parameters. Iteration proceeds until an objective-function, representing the least-square fit of model results and experimental data added to a regularization term, converges to a specified small value.

Bio-optical models [5] are employed to correlate the chlorophyll-a concentration to $a$ and $b$. At every iteration, the inverse solver generates a candidate solution that is a set of discrete chlorophyll-a concentration values. The RTE is solved by the Laplace transform discrete ordinate $\left(\mathrm{LTS}_{N}\right)$ method $[1,8,9]$. The associated optimization problem is solved by an Ant Colony System (ACS) [3] implementation. A intrinsic regularization scheme that pre-selects candidate solutions based on their smoothness is applied, quantified by a Tikhonov norm [7].

It is tested a hybrid inversion scheme, where the ACS solution provides an initial guess to the deterministic Levenberg-Marquardt (L-M) method. The spatial domain (geometrical depth) is discretized in a number of regions $(R)$. The coefficients and thus the chlorophyll-a concentration are assumed as being constant in each region. The dicrete chlorophyll-a profile is then defined by $(R+1)$ points.

In the case study, for a lower vertical resolution (ie, $R+1=11$ levels deep), ACS is not able to identify some details of the chlorophyll-a profile. Therefore, a higher resolution of the spatial domain is necessary to allow a correct identification of peaks of chlorophyll-a concentration.

Better ACS inversion results are then obtained using a higher resolution (ie, $R+1=81$ levels deep) in the discrete vertical domain. However, the following L-M inversion did not improve the 81 levels deep ACS result as well as the 11 levels deep ACS one. It is due to the increase of the ill-posed nature of this inverse problem at this higher resolution.

\section{RADIATIVE TRANSFER EQUATION IN HYDROLOGIC OPTICS}

The Radiative Transfer Equation (RTE) models the transport of photons through a medium. Light intensity is given by a directional quantity, the radiance $I$, that measures the 
rate of energy being transported at a given point and in a given direction. Considering a horizontal plane, this direction is defined by a polar angle $\mu$ (relative to the normal of the plane) and a azimuthal angle $\varphi$ (a possible direction in that plane). At any point of the medium, light can be absorbed, scattered or transmitted, according to the absorption $(a)$ and scattering (b) coefficients and to a scattering phase function that models how light is scattered in any direction. An attenuation coefficient $c$ is defined as $c=a+b$ and the geometrical depth is mapped to a optical depth $\tau$ that imbeds $c$. Assuming a plane-parallel geometry, and a single wavelength, the unidimensional integral-differential RTE, can be written as:

$$
\mu \frac{\partial}{\partial \tau} I(\tau, \mu, \varphi)+I(\tau, \mu, \varphi)=\frac{\varpi_{0}(\tau)}{4 \pi} \int_{-1}^{1} \int_{0}^{2 \pi} \beta\left(\mu, \varphi ; \mu^{\prime}, \varphi^{\prime}\right) I\left(\tau, \mu^{\prime}, \varphi^{\prime}\right) d \varphi^{\prime} d \mu^{\prime}+S(\tau, \mu, \varphi)
$$

where $\mu \in[-1,1]$ and $\varphi \in[0,2 \pi]$ are the cosine of the incident polar angle $\theta$ and the incident azimuthal angle, respectively. $\varpi_{0}(\tau)=b(\tau) / c(\tau)$ is the single scattering albedo. The scattering phase function $\beta\left(\mu, \varphi ; \mu^{\prime}, \varphi^{\prime}\right)$, gives the scattering beam angular distribution, mapping the incident beam direction $(\mu, \varphi)$ to the scattered direction $\left(\mu^{\prime}, \varphi^{\prime}\right)$, and the source term is $S(\tau, \mu, \varphi)$. The heterogeneous medium, in this case offshore ocean water is then modeled as a set of $R$ homogeneous finite layers. Boundary conditions are defined between regions, at the surface (incident light) and the bottom of the water. Each layer is denoted as being a region $r$ of the multiregion domain:

$$
\varpi_{0}(\tau)=\varpi_{r} \quad r=1,2, \ldots, R+1
$$

There are several resolution methods, most of them adopting the Chandrasekhar's decomposition on the azimuthal angle [2] that generates $L+1$ integral-differential equations, each one with no dependence on $\varphi$. For the discrete ordinate method, the above equations are approximated by a colocation method, where the $\mu$ integral is computed by the GaussLegendre quadrature formula. This yields a set of $N$ differential equations for each azimuthal mode. Each set (discretized RTE) is solved by the $\mathrm{LTS}_{N}$ method, that generates a system of equations of order $(R+1) \times N$. For the considered test cases, it was assumed $R=10$ and $R=80$, with $N=20$ and $L=0$ (azimuthal simmetry on $\varphi$ ).

This work employs a bio-optical models to correlate the absorption and scattering coefficients of each region to the chlorophyll concentration. These coefficients are assumed to be constant in each region. Therefore discrete values $a_{r}$ and $b_{r}$ can be estimated for each region from the discrete values $C_{r}$.

A bio-optical model was formulated by [6] for the absorption coefficient,

$$
a_{r}=\left[a^{w}+0.06 a^{c} C_{r}^{0.65}\right]\left[1+0.2 e^{-0.014(\lambda-440)}\right]
$$

where $a^{w}$ is the pure water absorption and $a^{c}$ is a nondimensional, statistically derived chlorophyllspecific absorption coefficient, and $\lambda$ is the considered wavelength, while another was $a_{g}^{w}$ formulated by [4] for the scattering coefficient,

$$
b_{r}=\left(\frac{550}{\lambda}\right) 0.30 C_{r}^{0.62}
$$




\section{INVERSION SCHEME}

This work formulates the inverse problem according to an implicit approach, leading to an optimization problem. the set of parameters to be estimated, is given by $\boldsymbol{p}$, in this case, the $R+1$ discrete values of the chlorophyll concentration $C$ at optical depths $\tau$ taken at the interface of the regions. Thus $p_{r}=C\left(\tau_{r}\right)$ for $r=0,1, \ldots, R$.

Experimental data are the discrete radiances $I\left(\tau_{r}, \mu_{i}\right)$ for $r=0,1, \ldots, R$ and $i=$ $1,2, \ldots, N$. The objective function $J(\boldsymbol{p})$ is given by the square difference between experimental and model radiances plus a regularization term:

$$
J(\boldsymbol{p})=\sum_{i=1}^{N} \sum_{r=0}^{R}\left[I^{\exp }\left(\tau_{r}, \mu_{i}\right)-I_{\boldsymbol{p}}\left(\tau_{r}, \mu_{i}\right)\right]^{2}+\gamma \Omega(\boldsymbol{p})
$$

The $R+1$ discrete values of the concentration are estimated from $(R+2) \times N$ radiance values. $\Omega(\boldsymbol{p})$ is the regularization function, that is weighted by a regularization parameter $\gamma$. For instance, the 2nd order Tikhonov regularization [13] is defined by

$$
\Omega[\boldsymbol{p}]=\sum_{i=2}^{R-1}\left(p_{i+1}-2 p_{i}+p_{i-1}\right)^{2} .
$$

The regularization term is required for noisy data due to the ill-posedness nature of inverse problems.

The influence of radiance data of higher depths can be underestimated since radiances decrease is nearly exponential with depth. Therefore, a depth correction factor may be used in the objective function.

It is used a depth correction factor $\left(C F_{r}\right)$, given by the ratio between the mean radiance $\overline{I_{r}}$ related to the polar angle $\mu$ at each level $r$ and the mean radiance at the surface $\overline{I_{1}}$. This is done separately for negative/upward $(u)$ and positive/downward $(d)$ polar directions. The depth correction factor for the regions $r=0,2, \ldots, R-1$ is given by:

$$
\begin{gathered}
\overline{I_{r}^{u}}=\sum_{i=1}^{N / 2} I^{\exp }\left(\tau_{r}, \mu_{i}\right) /(N / 2) \\
C F_{r+1}^{u}=\left(\overline{I_{1}^{u}} / \overline{I_{r}^{u}}\right)^{2} .
\end{gathered}
$$

For regions $r=1,2, \ldots, R$ the depth correction factor is given:

$$
\begin{gathered}
\overline{I_{r}^{d}}=\sum_{i=N / 2+1}^{N} I^{\exp }\left(\tau_{r}, \mu_{i}\right) /(N / 2) \\
C F_{r}^{d}=\left(\overline{I_{1}^{d}} / \overline{I_{r}^{d}}\right)^{2} .
\end{gathered}
$$

Thus, the objective function can be written as:

$$
\begin{aligned}
J(\boldsymbol{p})= & \sum_{i=1}^{N / 2} \sum_{r=0}^{R-1} C F_{r+1}^{u}\left[I^{e x p}\left(\tau_{r}, \mu_{i}\right)-I \boldsymbol{p}\left(\tau_{r}, \mu_{i}\right)\right]^{2}+ \\
& \sum_{i=N / 2+1}^{N} \sum_{r=1}^{R} C F_{r}^{d}\left[I^{\exp }\left(\tau_{r}, \mu_{i}\right)-I_{\boldsymbol{p}}\left(\tau_{r}, \mu_{i}\right)\right]^{2}+\gamma \Omega(\boldsymbol{p})
\end{aligned}
$$




\section{NUMERICAL RESULTS}

A experimental data of the chlorophyll-a concentration was measured on the Brazilian coast using a sensor for natural fluorescence (PNF, Biospherical instruments), on board of the oceanographic campaing at the fall 2003 [10]. The radiative transfer problem is considering $N=20$ polar angles and $L=0$ (azimuthal symmetry). In this test case, synthetic data was used to simulate the experimental radiance values.

Since radiance decayment is nearly exponential with depth, the use of a depth correction factor may be required: radiances near the surface have a greater influence in the objective function than those at higher depths. The depth correction factor, shown in Section 3, is used to balance the radiance values. The set of radiance data was corrupted with $5 \%$ gaussian noise.

The inverse solver, based on a ACS implementation, was tested for this offshore ocean water. Instead of the classical regularization, the smoothness-based pre-selection was employed in the generation of the ants. The 2 nd order Tikhonov norm was used as smoothness criteria.

Figure 1(a) shows the inverse solution obtained from a realization of the ACS for a 11depths vertical resolution profile (called $\mathrm{ACS}_{11}$ ). It was not able to identify the two peaks of the exact chlorophyll-a profile. As is illustrated in Figure 1(b), a improved solution is reached applying the Levenberg-Marquardt deterministic method (called L-M $\mathrm{M}_{11}$ ), with $\mathrm{ACS}_{11}$ solution as its initial guess.

However, although the solution in each 11 depths have values very close to exact solution, the amount of points adopted to form the vertical profile of the solution is not enough to identify two peaks of concentration.

It is necessary to increase the vertical resolution of the profile for a number of points in depth which makes it possible to identify these two maxima of concentration.

As an alternative to obtain a solution with enhanced resolution, we decided to recover the concentration values of chlorophyll-a in each meter of the profile depth, ie, in 81 levels deep.

Two strategies were employed to achieve it. In the first, was employed again the hybrid scheme of inversion, where the solution $\mathrm{ACS}_{11}$ is interpolated to 81 levels (called $\mathrm{AACS}_{81}$ ) and enter as initial guess to the Levenberg-Marquardt method (called L-M $\mathrm{M}_{81}$ ).

In the second strategy, was used as the initial guess for $\mathrm{L}_{-} \mathrm{M}_{81}$ the solution already obtained with Levenberg-Marquardt in 11 levels of depth, interpolated into 81 levels (called $\left.\mathrm{iL}_{-} \mathrm{M}_{81}\right)$

It is observed in Figures 2(a) e 2(b) that none of these strategies has produced a result much better than the ACS initial guess. In fact, the second maximum concentration remains omitted, while the first maximum is poorly recovered and, in addition to that, the whole profile becomes more irregular.

This is due precisely to increase the number of points to be estimated. The inverse problem becomes increasingly ill-posed with the increase in the number of profile depths to be recovered, and the LM method implemented does not use any regularization, as the one used in ACS method.

A new alternative was tried to obtain a solution in 81 levels with the ACS (called $\mathrm{ACS}_{81}$ ), using then this result as initial guess to the L-M. At this higher resolution, as seen in 
Figure 3(a) the result obtained in $\mathrm{ACS}_{81}$ was able to identify the two maximum concentration with reasonable accuracy. However, Figure 3(b) shows that $\mathrm{LM}_{81}$ was not able to find the exact position of these peaks, as could be expected.

\section{FINAL REMARKS}

This work presented a methodology for the estimation of an enhanced vertical resolution profile of chlorophyll-a concentration in natural waters, using radiance data in different depths.

The ACS-based inverse solver yielded a initial estimation of chlorophyll vertical profiles using a depth correction factor for the radiances data in the given test case for radiance data with 5\% noise. No standard regularization was used in the objective function besides the pre-regularization scheme.

Whereas a vertical resolution of 11 levels deep, the hybrid approach inversion (Ant Colony System + Levenberg-Marquardt) was not able to identify two existing maximum concentration of chlorophyll-a of the exact profile.

It was therefore necessary to adopt a vertical resolution with 81 points, one for each meters in depth, in order to enable the identification of the peaks concentration points. In this context, the ACS method itself can recover with reasonable accuracy all the points of the profile.

It was expected that the subsequent use of the L-M method could provide a more exact position of the concentration peaks. But due to increasily ill-posed nature of the problem, this goal was not achieved.

In future work, we plan to evaluate the feasibility of adopting a strategy of gradual increase of vertical resolution, where the result of the previous resolution points are frozen by varying only the new points of the current higher resolution. 


\section{REFERENCES}

[1] Barichello L. B., Vilhena M. T., "A general approach to one-group one-dimensional transport equation”. Kerntechnik 58, 128-184, 1993.

[2] Chandrasekhar S, "Radiative transfer". Dover, New York, 1960.

[3] Dorigo M., Maniezzo V., Colorni A., "The ant system: optimization by a colony of cooperating agents". IEEE Transactions on Systems, Man, and Cybernetics? Part B 26, 29-41, 1996.

[4] Gordon H. R., Morel A., "Remote assessment of ocean color for interpretation of satellite visible imagery, a review". In Lectures Notes on Coastal Estuarine Studies, 114, SpringerVerlag, New York, 1983.

[5] Mobley C. D., "Light and water: radiative transfer in natural waters". Academic Press, San Diego, 1994.

[6] Morel A., " Light and marine photosynthesis: a spectral model with geochemical and climatological implications.”. Progress in Oceanography, 26, 263?306, 1991.

[7] Preto A. J., CamposVelho H. F, Becceneri, J.C., Arai, N. N., Souto R. P., Stephany S., "A new regularization technique for an ant-colony based inverse solver applied to a crystal growth problem". In Proceedings Inverse Problem in Engineering Seminar, 147-153, Cincinnati, 2004.

[8] Segatto C. F., Vilhena M. T., "Extension of the ltsn formulation for discrete ordi- nates problem without azimuthal symmetry". Annals of Nuclear Energy, 21, 701?710, 1994.

[9] Segatto C. F., Vilhena M. T., Gomes M. G., "The one-dimensional ltsn solution in a slab with high degree of quadrature". Annals of Nuclear Energy, 26, 925?934, 1999.

[10] Souto R. P., Kampel M., Brandini F., CamposVelho H. F., Stephany S., "Análise comparativa de medições in situ e orbital de radiâncias do oceano na estimativa de concentração de cloro?la-a.”. In Anais eletrônicos: Simpósio Brasileiro de Sensoriamento Remoto, 3679?3686, São José dos Campos, 2005.

[11] Souto R. P., Campos Velho H. F., Stephany S, Kampel M., "Chlorophyll concentration profiles from in situ radiances by ant colony optimization". Journal of Physics: Conference Series , 124, 012047, 2008.

[12] Tao Z., McCormick N. J., Sanchez R., "Ocean source and optical property estimation from explicit and implicit algorithms". Applied Optics , 33, 3265?3275, 1994.

[13] Tikhonov A. N., Arsenin V. S., "Solutions of Ill-posed problems". V.H. Winston and Sons, Washington, 1977. 


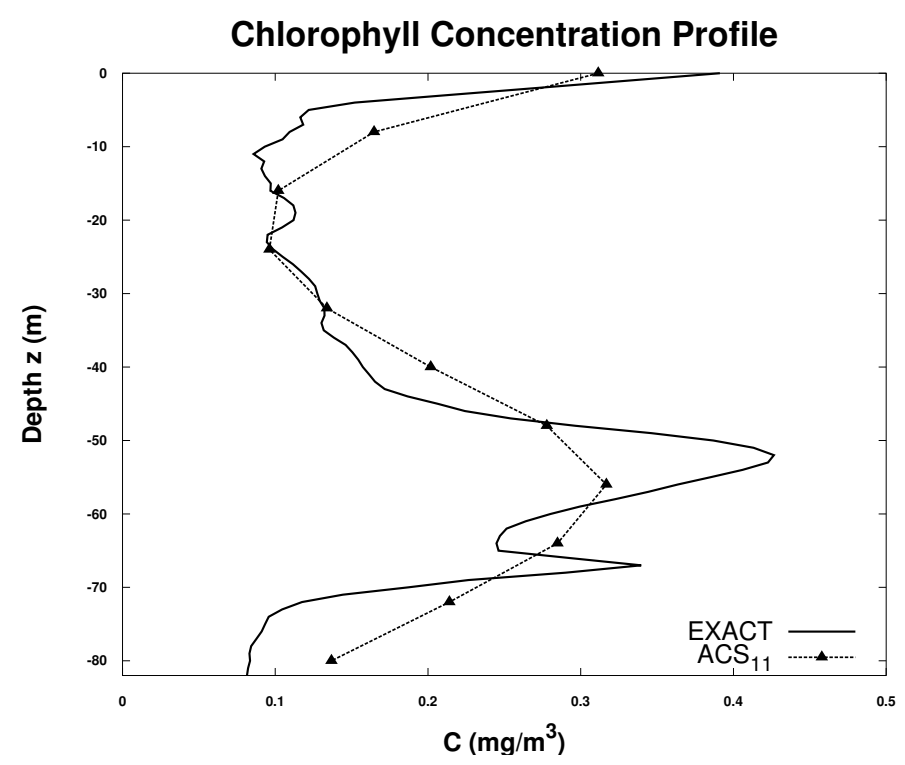

(a)

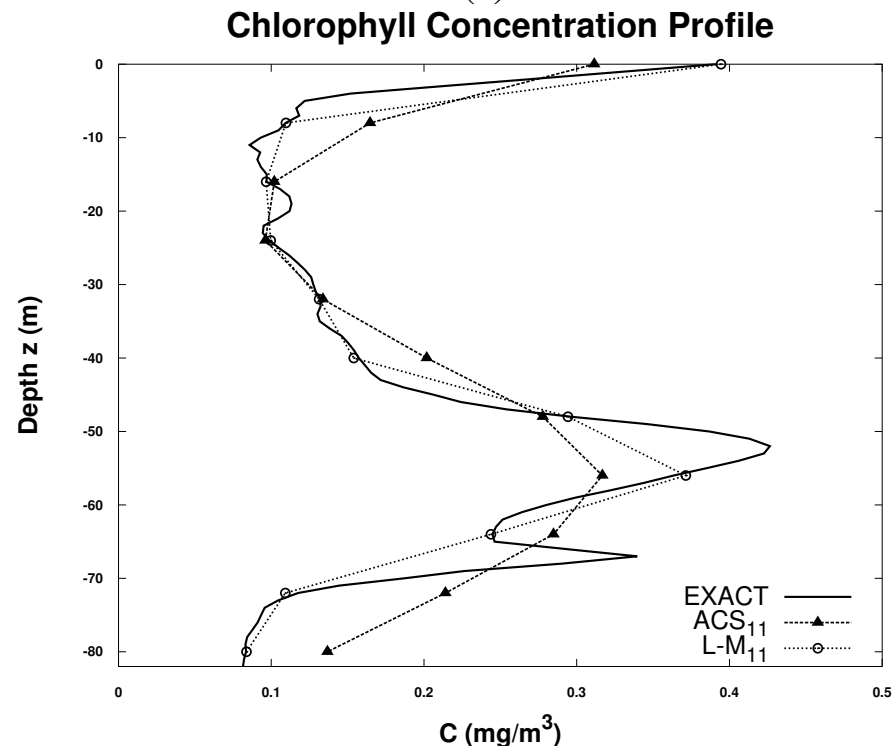

(b)

Figura 1. Reconstruction of the 11 depths chlorophyll-a vertical profile:

(a) obtained with Ant Colony System $\left(\mathrm{ACS}_{11}\right)$

(b) obtained with Levenberg-Marquardt $\left(\mathrm{L}-\mathrm{M}_{11}\right)$, with $\mathrm{ACS}_{11}$ as initial guess. 


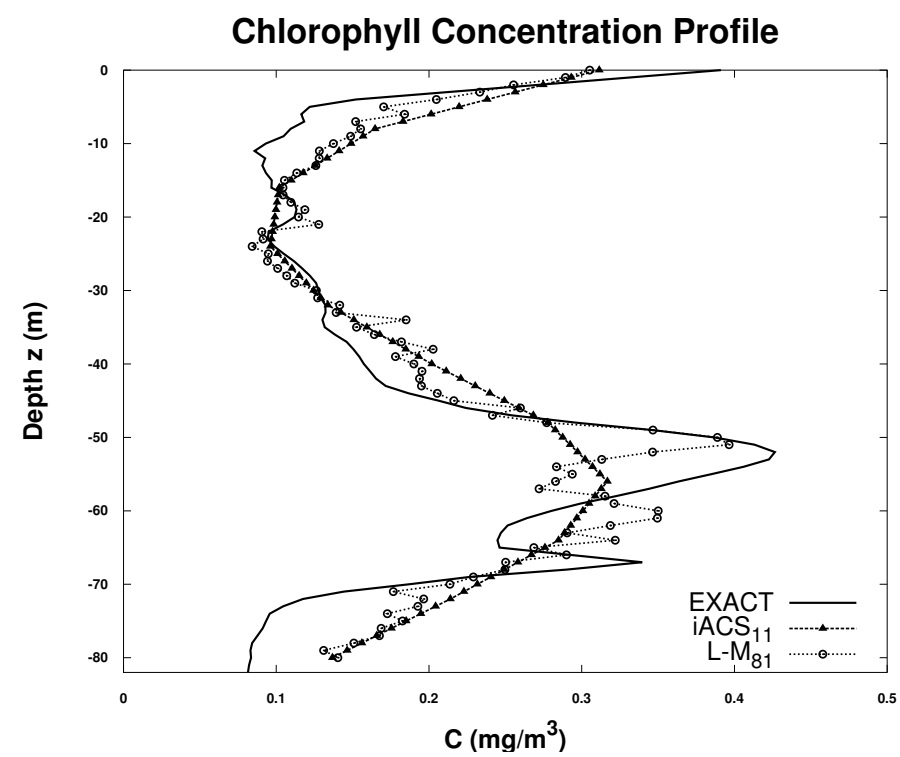

(a)

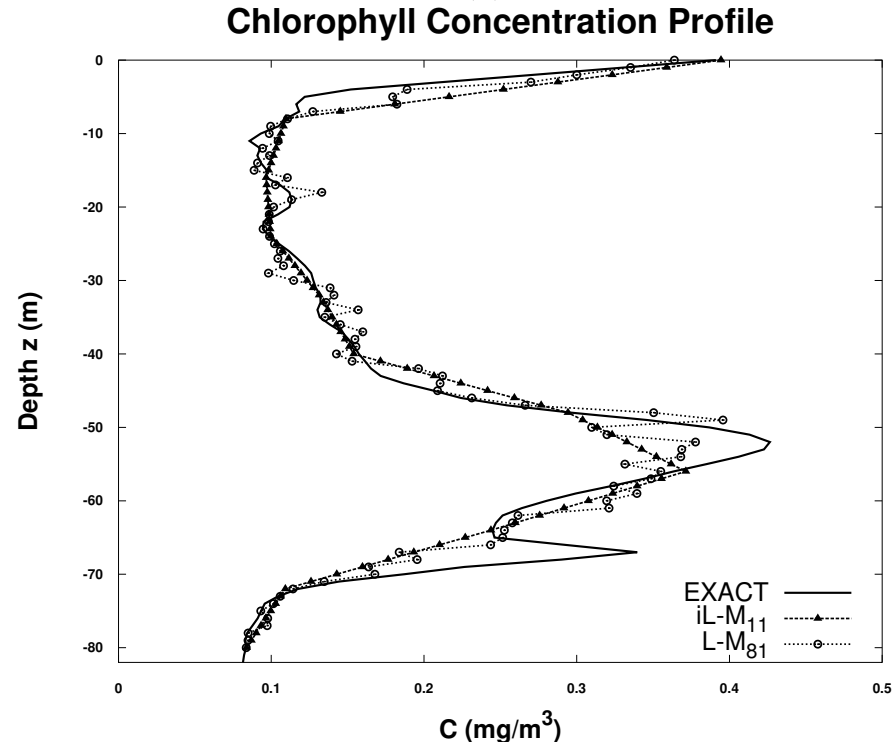

(b)

Figura 2. Reconstruction of the 81 depths chlorophyll-a vertical profile obtained with Levenberg-Marquardt (L-M $\left.\mathrm{M}_{81}\right)$ :

(a) with interpolated $\mathrm{ACS}_{11}$ as initial guess (iACS ${ }_{11}$ )

(b) with interpolated $\mathrm{L}-\mathrm{M}_{11}$ as initial guess (iL-M $\mathrm{M}_{11}$ ). 


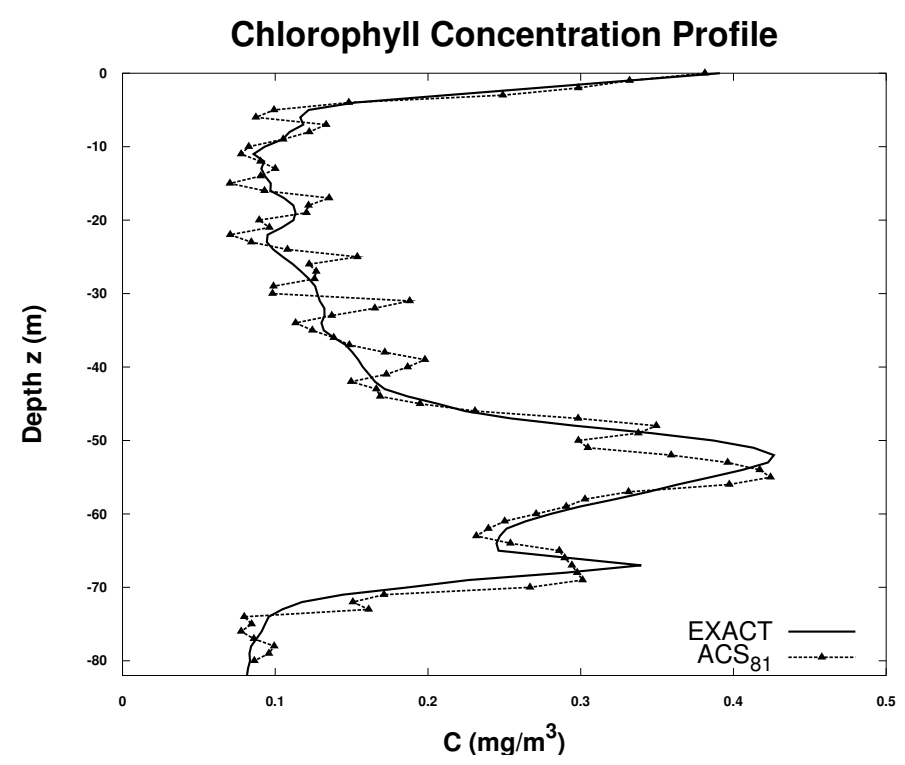

(a)

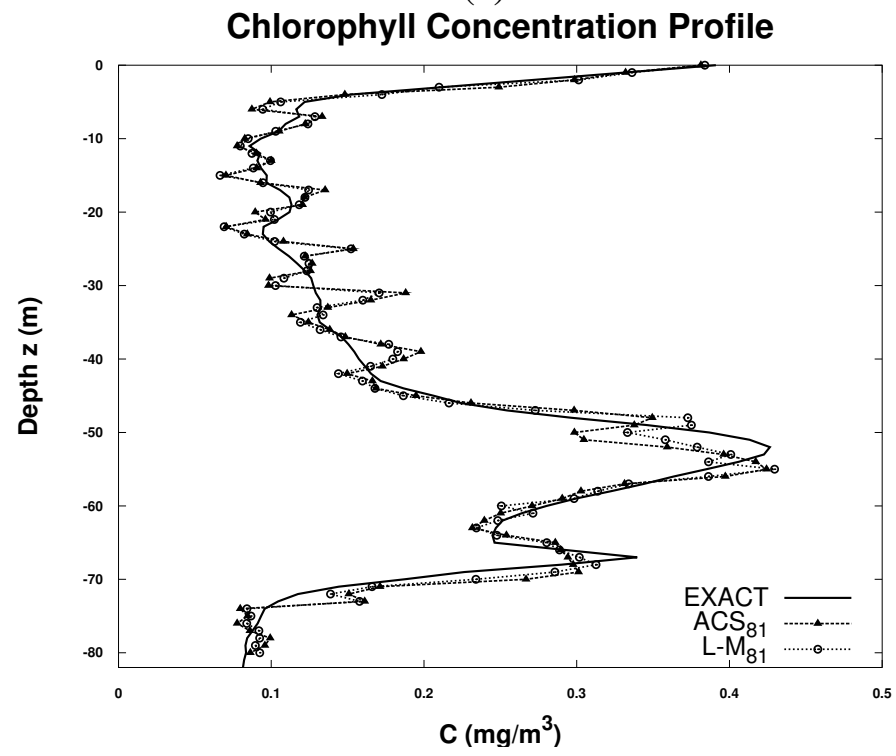

(b)

Figura 3. Reconstruction of the 81 depths chlorophyll-a vertical profile:

(a) obtained with Ant Colony System $\left(\mathrm{ACS}_{81}\right)$

(b) obtained with Levenberg-Marquardt $\left(\mathrm{L}-\mathrm{M}_{81}\right)$, with $\mathrm{ACS}_{81}$ as initial guess. 